microbubbles: safety and efficacy evaluation in rhesus macaques. Cancer Res 2012;72:3652-63

7. McDannold N, Clement GT, Black P, et al. Transcranial magnetic resonance imaging-guided focused ultrasound surgery of brain tumors: initial findings in 3 patients. Neurosurgery 2010;66:323-32

8. Jeanmonod D, Werner B, Morel A, et al. Transcranial magnetic resonance imaging-guided focused ultrasound: noninvasive central lateral thalamotomy for chronic neuropathic pain. Neurosurg Focus 2012;32:E1

9. Lipsman N, Schwartz ML, Huang Y, et al. MR-guided focused ultrasound thalamotomy for essential tremor: a proof-of-concept study. Lancet Neurol 2013;12:462-68

10. Elias WJ, Huss D, Voss T, et al. A pilot study of focused ultrasound thalamotomy for essential tremor. $N$ Engl J Med 2013;369:640-48

11. InSightec. A Feasibility Study to Evaluate Safety and Initial Effectiveness of ExAblate Transcranial MR Guided Focused Ultrasound for Unilateral Thalamotomy in the Treatment of Medication-Refractory Tremor Dominant Idiopathic Parkinson's Disease. In: ClinicalTrials.gov. Bethesda, Maryland: National Library of Medicine (US). 2000 [cited 2013 Sept 1]. http:// clinicaltrials.gov/ct2/show/NCT01772693. 2013

12. Burgess A, Ayala-Grosso CA, Ganguly M, et al. Targeted delivery of neural stem cells to the brain using MRI-guided focused ultrasound to disrupt the blood-brain barrier. PLoS One 2011;6:e27877

13. Jordao JF, Thevenot E, Markham-Coultes K, et al. Amyloid-beta plaque reduction, endogenous antibody delivery and glial activation by brain-targeted, transcranial focused ultrasound. Exp Neurol 2013;248C:16-29

14. Arvanitis CD, Livingstone MS, McDannold N. Combined ultrasound and MR imaging to guide focused ultrasound therapies in the brain. Phys Med Biol 2013;58:4749-61

15. Saqqur M, Tsivgoulis G, Nicoli F, et al. The role of sonolysis and sonothrombolysis in acute ischemic stroke: a systematic review and meta-analysis of randomized controlled trials and case-control studies. J Neuroimaging 2013 Apr 22. [Epub ahead of print]

16. Monteith SJ, Harnof S, Medel R, et al. Minimally invasive treatment of intracerebral hemorrhage with magnetic resonance-guided focused ultrasound. J Neurosurg 2013;118:1035-45

17. Yang HW, Hua MY, Hwang TL, et al. Non-invasive synergistic treatment of brain tumors by targeted chemotherapeutic delivery and amplified focused ultrasound-hyperthermia using magnetic nanographene oxide. Adv Mater 2013;25:3605-11

18. Martin E, Jeanmonod D, Morel A, et al. High-intensity focused ultrasound for noninvasive functional neurosurgery. Ann Neurol 2009;66:858-61

19. Yamada $K$, Akazawa $K$, Yuen S, et al. MR imaging of ventral thalamic nuclei. AJNR Am J Neuroradiol 2010;31:732-35

EDITORIAL

\section{Simple MRI Metrics Contribute to Optimal Care of the Patient with Multiple Sclerosis}

\author{
J.H. Simon, R.A. Bermel, and R.A. Rudick
}

M $\mathrm{R}$ imaging has been a critical element in multiple sclerosis care because it has been the basis, along with clinical measures, for testing treatment efficacy. MR imaging serves as a pri-

Indicates article with supplemental on-line tables.

http://dx.doi.org/10.3174/ajnr.A3937 mary outcome measure in phase II and a secondary outcome measure in phase III clinical trials in MS. ${ }^{1}$ There are now 10 approved MS disease-modifying drugs, all showing measureable impact in population studies on inflammatory disease as indicated by new T2 hyperintense and/or gadolinium-enhancing lesions on MR imaging. MR imaging initially impacted the field as an important component of diagnostic criteria, ${ }^{2}$ in part because MR imaging is much more sensitive to early MS than are clinical features. For similar reasons, clinicians have embraced the practice of monitoring subclinical MR imaging activity for treatment decisions, though formal criteria for an actionable response to $\mathrm{MR}$ imaging activity in an individual patient have been limited (Online Table 1). MR imaging monitoring is also critical for detecting complications of therapy-for example, infection (progressive multifocal leukoencephalopathy) or inflammation (immune reconstitution inflammatory syndrome). ${ }^{3}$

Several recent initiatives by the MS community have addressed the concept of individualized, more tailored, and sometimes more aggressive early treatment. Treatment escalation has only recently become feasible with the introduction of new, potentially stronger MS treatments based on differing mechanisms and molecular targets. ${ }^{4}$ As a result, MR imaging activity will be increasingly used in clinical practice to determine whether patients are responding to treatment or may benefit from a change in treatment or escalation to higher-risk therapy (On-line Table 1). For example, the Canadian MS Working Group guidelines were updated in $2013,{ }^{5}$ on the basis of combinations of relapse, disability, and MR imaging scores, for recommendations classified as low, medium, or high concern. The Rio score, developed in Barcelona, was modified recently on the basis of a validation study to include only MR imaging activity and relapse indicators. ${ }^{6}$ Enhancing lesions, followed by relapses and new T2 lesions during the initial 2 years, were the best predictors of disability 15 years later in treated (distinct from placebo) patients in the interferon (IFN) $\beta$-1a trial, ${ }^{7}$ suggesting that persistent inflammatory disease activity in patients on IFN reflected nonresponse to therapy. An analysis by Dobson et $\mathrm{al}^{8}$ from 11 studies with IFN- $\beta$ treatment found that those who develop new MR imaging lesions on IFN- $\beta$ within 2 years of starting therapy are at significantly higher risk of future relapses and/or disability worsening and that these patients can be identified after just $6-12$ months of treatment.

The simple MR imaging measures of focal T2 hyperintense and enhancing lesions seem to contribute strongly to relapse and disability outcomes and contribute significantly to brain atrophy, a surrogate of disability. This association is highlighted in a recent meta-analysis by Sormani et al, ${ }^{9}$ based on $>13,500$ patients with relapsing MS in 13 clinical trials. The correlation coefficients $\left(R^{2}\right)$ with downstream disability for new/enlarging T2 lesions and brain atrophy were 0.61 and 0.48 , respectively, with both measures retained in a final model with a combined $R^{2}$ of 0.75 , strongly supporting the use of these MR imaging outcomes as clinical surrogate measures when applied in an appropriate clinical-/treatment-specific context. ${ }^{9}$

It is likely that in the future, advanced quantitative and functional measures by MR imaging will assume far greater impor- 
tance in measuring aspects of neurodegeneration, de- and remyelination, and particularly in progressive stages of MS, including in individual patients. However, the currently recognized success of the existing MS therapies is thought to be predominantly based on the impact on the early inflammatory stages of disease, with a variable and lesser, perhaps only secondary, impact on neurodegeneration. Standardized brain volume (atrophy) measures are predictive of disability and, when applied serially, can be used to assess atrophy patterns, including in individual patients, ${ }^{10}$ but these are not widely available or currently thought to be practical in clinical practice unless and until MR imaging manufacturers or other third parties support these measures.

There is an emerging consensus in the MS field that successful treatment results in no evident inflammatory disease activity (NEIDA), defined as the absence of new relapses or new MR imaging lesions. This was discussed at a recent international consensus workshop sponsored by the Cleveland Clinic (Las Vegas; December 12-14, 2013). Standardized MR imaging lesion reporting was identified by survey as a critical element for future implementation and testing of NEIDA. There have been prior initiatives to improve MS clinical care through standardized requests, MR imaging acquisition, and interpretation templates, including by the Consortium of MS Centers. ${ }^{11}$ More recent revisions recognize the improved hardware, including the potential shift to a $3 \mathrm{D}$ acquisition technique (see www.mscare.org). MR imaging technology evolves rapidly, field strength is ever-increasing, and acquisition and processing techniques will continue to impact lesion counts, which will necessarily evolve as well. Nevertheless, well-planned $2 \mathrm{D}$ and $3 \mathrm{D}$ acquisition techniques and attention to detail in manual or computerized registration will provide a basis for accurate serial analyses.

While there is almost certain to be disagreement as to the optimal cut-point for lesion counts to support treatment for MS, standardized, high-quality MR imaging acquisition combined with reports that provide the essential elements for MS diagnosis or therapeutic decision-making (On-line Tables 2 and 3) can, no doubt, improve outcomes for patients with MS.

Disclosures: Jack H. Simon—UNRELATED: Consultancy: Cleveland Clinic Foundation, Comments: MS Experts Consensus Summit: No Evidence of Disease Activity as a Treatment Target in MS, Biogen Idec, Comments: MS Clinical Research Plan Advisory Board, Grants/Grants Pending: Biogen Idec, ${ }^{*}$ Comments: CHAMPS/CHAMPIONS MS Trial research support (none current), manuscript preparation (editorial) support (no funds involved), Kinkel et al and Simon et al manuscripts of CHAMPS/CHAMPIONS outcomes, Payment for Manuscript Preparation: Biogen Idec, Comments:
Manuscript preparation support continues (no money involved), Royalties: Cambridge University Press, Comments: Co-Editor of Imaging Acute Neurologic Disease (no royalties to date), Travel/Accommodations/Meeting Expenses Unrelated to Activities Listed: National Institutes of Health/National MS Society, ${ }^{*}$ Canadian MS Society, ${ }^{*}$ Guthy Jackson Foundation, ${ }^{*}$ Comments: Study Section Work: National Institutes of Health/National MS Society/Canadian, no personal fees or honoraria accepted, travel reimbursement only (National Institutes of Health/National MS Society), Canadian MS/Italian MS/Ad Hoc (University of Washington Foundation), no fees or travel funds involved. Robert A. Bermel—RELATED: Grant: Biogen Idec,* Genzyme, ${ }^{*}$ Comments: unrestricted educational grant in support of the 2014 No Evidence of Inflammatory Disease Activity as a Treatment Target Continuing Medical Education meeting, Consultancy: Biogen Idec, Novartis, Genzyme, Questcor, Astellas, Comments: in compliance with institutional conflict of interest policies, Grants/Grants Pending: Novartis. * Richard A. Rudick-UNRELATED: Consultancy: Biogen Idec, Novartis, Genzyme, Comments: consulting on MS drugs, Grants/ Grants Pending: Novartis, ${ }^{*}$ Genzyme, ${ }^{*}$ Comments: grants to the Cleveland Clinic Foundation. *Money paid to the institution.

\section{REFERENCES}

1. Barkhof F, Simon JH, Fazekas F, et al. MRI monitoring of immunomodulation in relapse-onset multiple sclerosis trials. Nat Rev Neurol 2012;8:13-21

2. Polman CH, Reingold SC, Banwell B, et al. Diagnostic criteria for multiple sclerosis: 2010 revisions to the McDonald criteria. Ann Neurol 2011;69:292-302

3. Yousry TA, Pelletier D, Cadavid D, et al. Magnetic resonance imaging pattern in natalizumab-associated progressive multifocal leukoencephalopathy. Ann Neurol 2012;72:779-87

4. Kantarci OH, Pirko I, Rodriguez M. Novel immunomodulatory approaches for the management of multiple sclerosis. Clin Pharmacol Ther 2014;95:32-44

5. Freedman MS, Selchen D, Arnold DL, et al. Treatment optimization in MS: Canadian MS Working Group updated recommendations. Can J Neurol Sci 2013;40:307-23

6. Sormani MP, Rio J, Tintore M, et al. Scoring treatment response in patients with relapsing multiple sclerosis. Mult Scler 2013;19:605-12

7. Bermel RA, You X, Foulds P, et al. Predictors of long-term outcome in multiple sclerosis patients treated with interferon beta. Ann Neurol 2013;73:95-103

8. Dobson R, Rudick RA, Turner B, et al. Assessing treatment response to interferon- $\boldsymbol{\beta}$ : is there a role for MRI? Neurology 2014; 21;82:248-54

9. Sormani MP, Arnold DL, De Stefano N. Treatment effect on brain atrophy correlates with treatment effect on disability in multiple sclerosis. Ann Neurol 2014;75:43-49

10. Fisher E, Lee, JC, Rudick, R. Temporal patterns of brain atrophy in individual multiple sclerosis patients. The American Academy of Neurology's 65th AAN Annual Meeting in San Diego, CA; March $16-23,2013$

11. Simon JH, Li D, Traboulsee A, et al. Standardized MR imaging protocol for multiple sclerosis: Consortium of MS Centers Consensus Guidelines. AJNR Am J Neuroradiol 2006;27:455-61 